\title{
Analysis and Simulation of Solidification for Phase Change Materials in a Cylindrical Container
}

\author{
H.C. Tien \\ Department of Marine and Mechanical Engineering National Taiwan Ocean University Keelung, Taiwan, R.O.C. \\ J.S. Chang \\ Department of Marine and Mechanical Engineering National Taiwan Ocean University Keelung, Taiwan, R.O.C.
}

Follow this and additional works at: https://jmstt.ntou.edu.tw/journal

Part of the Mechanical Engineering Commons

\section{Recommended Citation}

Tien, H.C. and Chang, J.S. (1993) "Analysis and Simulation of Solidification for Phase Change Materials in a Cylindrical Container," Journal of Marine Science and Technology. Vol. 1: Iss. 1, Article 8.

DOI: $10.51400 / 2709-6998.2478$

Available at: https://jmstt.ntou.edu.tw/journal/vol1/iss1/8

This Research Article is brought to you for free and open access by Journal of Marine Science and Technology. It has been accepted for inclusion in Journal of Marine Science and Technology by an authorized editor of Journal of Marine Science and Technology. 


\title{
ANALYSIS AND SIMULATION OF SOLIDIFICATION FOR PHASE CHANGE MATERIALS IN A CYLINDRICAL CONTAINER
}

\author{
H.C. Tien and J.S. Chang \\ Department of Marine and Mechanical Engineering \\ National Taiwan Ocean University \\ Keelung, Taiwan, R.O.C.
}

Key words: solidification, phase change materials, enthaply formulation, thick-walled container

\begin{abstract}
The objective of this paper is to study the solidification problem of the phase change materials (PCM) in a thick-walled cylindrical container. Through varying each controlling parameter such as the thermophysical properties of wall (mold), wall thickness, aspect ratio of the container, initial temperature of PCM and the external cooling conditions, etc., the effect of each parameter on the solidification process is shown clearly. By knowing how a thick wall interacts with such a phase change phenomenon, it is therefore possible to well control the solidification process and also to improve the solid structure formed.

In this study, convective boundary conditions are imposed on the outer and bottom boundaries for simulating real ambient cooling conditions. The top surface is kept insulated. The mathematical model of this study is based on the enthalpy formulation. An alternating-directionimplicit (ADI) scheme is employed to solve the pertinet governing equations along with the boundary conditions. Due to the fact that the PCM thermal properties are different from the thermal properties of the wall, the well-known harmonic mean method is used to resolve the discontinuity of the conductivity across the interface. It is demonstrated in this study that the effects of the thermal properties of the wall and the external cooling conditions on the solidification process are quite significant. Furthermore, it is found that the controlling parameters which include the wall thickness, container aspect ratio and the initial temperature for PCM have a direct influence on the solidification rate. The results extracted from this investigation can be applied for many similar solidification problems.
\end{abstract}

\section{INTRODUCTION}

Solid/liquid phase change problems have been studied quite extensively due to broad applications. These applications include energy storage, metal casting, welding, metallurgy and purification of metals etc. The solidification process, in general, is greatly affected by the thermophysical properties and initial temperature of the wall (mold) as well as cooling conditions. When either melting or solification occurs, latent heat is absorbed or released together with such phase change process. Viskanta [19] pointed out clearly that one of the main issues in melting/ solidification problems is to well control the absorption or release rate of the latent heat .

In this paper, the solidification problem for a phase change material (PCM) in a thick-walled cylindrical container is investigated. The fusion temperature of the PCM is a single value rather than a temperature range. Similar problems were studied in [15, 
17, 18]. However in Ref. [15], convective boundary conditions were not taken into account. Besides, the initial temperature was assumed to be at the fusion temperature and therefore the "superheat" effects were not considered in Ref. [15] either, In the present study, the initial temperature is either at the fusion temperature or above; that is, "superheat" is taken into account. However, natural convection is not included in this study since its effects were found to be not so significant in such inward solidification problems [16]. The influence of the fluid motion on the solidification process was discussed at length in $[5,6]$. The main objective of this study is to analyze the solidification problem for a PCM in a thick-walled cylindrical container. The effects of each controlling parameter such as the thickness and thermophysicl properties of the wall, the aspect ratio of the container, the initial temperature of the PCM and the external cooling conditions are investigated in detail.

In this study, the enthalpy method instead of the temperature-based mehtod is adopted to express the governing energy equations. This is due to the fact that both the liquid phase and solid phase energy equations can be combined into one with the use of enthalpy method and hence there is no need to track the interface. In addition, a fixed grid system can be employed associated with any appropriate numerical scheme. Previous studies using the enthalpy formulation can be exemplified as $[3,4,7,14]$. The derivation of the enthalpy energy equation can be found in [14]. It should be noted that the liquid PCM solidifies in a thick-walled container and the thermophysical properties of the wall are generally different from that of the PCM. In order to resolve such difficulty, the harmonic mean procedure proposed by Patankar [8] is employed.

\section{ANALYSIS}

\section{Problem Statement}

The liquid phase change material (PCM) is initially at the fusion temperature or above. The cylindrical container (mold) is thick-walled. As shown in Fig. 1, the outer radius and the height of the container are, separately, $R$ and $Z$. The wall thickness in the radial direction is $R_{w}$, and the wall thickness in the vertical direction is $Z_{w}$. As for the boundary conditions, the left boundary is adiabatic due to symmetry and the top boundary is assumed to be insulated in this study. The other two boundaries are subjected to convective boundary conditions for simulating the real ambient cooling conditions. It is well known that the convective boundary conditions are more general than the isothermal as well as the adiabatic boundary conditions. When the Biot number approaches infin

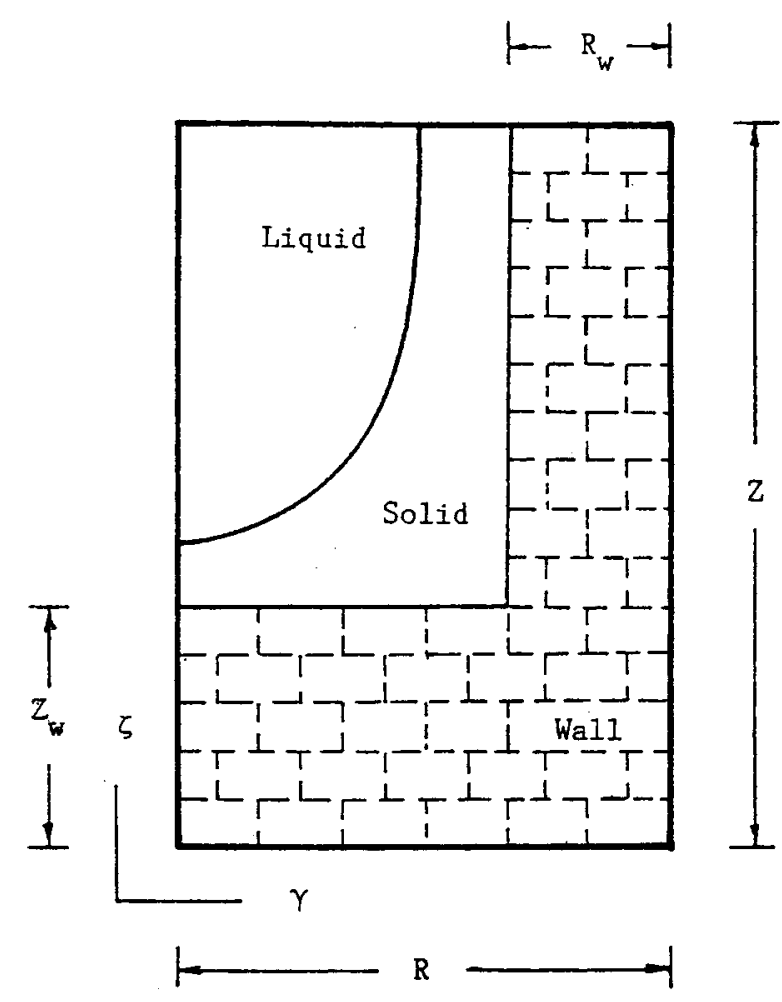

Fig. 1. The cross section of the container.

ity, the convective boundary condition gets close to the isothermal condition. On the other hand, the convective boundary condition reduces to the adiabatic condition as the Biot number approaches zero. The effects of the Biot number on the solidification process will be discussed in detail in this paper. It is noted that the initial temperature of the wall is set to be equal to the ambient temperature $T_{\infty}$.

\section{Mathematical Formulation}

Based on the enthalpy formulation in [14], the axisymmetric heat conduction equation accounting for phase change in cylindrical coordinate system can be obtained as

$$
\rho \frac{\partial h}{\partial t}=\frac{k}{\gamma} \frac{\partial}{\partial \gamma}\left(\gamma \frac{\partial T}{\partial \gamma}\right)+k \frac{\partial^{2} T}{\partial \zeta^{2}}
$$

In equation (1), $h$ and $T$ denote the enthalpy and the temperature, respectively. It should be mentioned that $h$ is the sum of the sensible heat and the latent heat. The nondimensional variables and parameters associated with the present problem can be defined as follows.

$$
\bar{G}=\frac{R}{Z} ; \quad r=\frac{\gamma}{R} ; \quad z=\frac{\zeta}{R}=\frac{\zeta}{(Z G)}
$$




$$
\begin{aligned}
& \theta=\frac{h-h_{f r}}{\lambda} ; \quad \bar{\alpha}=\frac{\alpha_{w}}{\alpha} ; \quad S_{t e}=\frac{T_{f r}-T_{r e f}}{\lambda / c} \\
& \bar{r}=\frac{R_{w}}{R} ; \quad \bar{k}=\frac{k_{w}}{k} ; \quad F_{o}=\frac{k t}{\rho c R^{2}} \\
& \hat{c}=\frac{c}{c_{L}} ; \quad \bar{Z}=\frac{Z_{w}}{Z} ; \quad \phi=\frac{T-T_{f r}}{\lambda_{c}}
\end{aligned}
$$

The energy equation, after nondimensionlization, assumes the form

$$
\frac{\partial \theta}{\partial F_{o}}=\frac{\partial^{2} \phi}{\partial r^{2}}+\frac{1}{r} \frac{\partial \phi}{\partial r}+\frac{\partial^{2} \phi}{\partial r^{2}}=\nabla^{2} \phi
$$

where $\theta$ and $\phi$ are the nondimensional enthalpy and temperature respectively, and their definitions can be found in equation (2).

There are three regions in the PCM domain, namely, liquid region, solid region and fusion area. The relationships between the enthalpy and the temperature are stated below:

(a) solid region

$$
T<T_{f r} ; h=c T
$$

(b) fusion area

$$
T=c T_{f r} ; c T_{f r} \leq h \leq \lambda+c T_{f r}
$$

(c) liquid region

$$
T>T_{f r} ; h=\lambda+c T_{f r}+c_{L}\left(T-T_{f r}\right)
$$

In nondimensional form, equations (4)-(6) can be expressed in terms of the dimensionless enthalpy $\theta$ and the dimensionless temperature $\phi$ as

(a) solid region

$$
\phi<0 ; \theta=\phi
$$

(b) fusion area

$$
\phi=0 ; 0 \leq \theta \leq 1
$$

(c) liquid region

$$
\phi>0 ; \theta=1+\frac{\phi}{\hat{c}}
$$

The dimensionless energy equation for the wall can be obtained similarly, as

$$
\frac{\partial \theta}{\partial F_{0}}=\bar{\alpha} \cdot \nabla^{2} \phi
$$

where $\bar{\alpha}=\alpha_{w} / \alpha$ is the ratio of the thermal diffusivity of the wall to the thermal diffusivity of the solid PCM. The relationship between the enthalpy and temperature of the wall is

$$
h=c_{w} T
$$

Equation (11) can be also written in dimensionless form as

$$
\theta=\phi
$$

\section{Numerical Solution}

Consider a representative PCM control volume shown in Fig. 2. Based on this control volume, the energy equation, equation (3), can be transformed into a finite difference equation after discretization, which is

$$
\begin{aligned}
\frac{\theta_{P}^{k}-\theta^{k-1}{ }_{P}}{\Delta F_{O}} & =\left(A_{N} \phi^{k}{ }_{N}+A_{P} \phi_{P}^{k}+A_{S} \phi_{S}^{k}\right) \\
& +\left(B_{E} \phi_{E}^{k}+B_{P} \phi_{P}^{k}+B_{W} \phi^{k}{ }_{W}\right)
\end{aligned}
$$

where

$$
\begin{aligned}
& A_{N}=\frac{1}{(\Delta z)^{2}} ; A_{S}=\frac{1}{(\Delta z)^{2}} \\
& A_{P}=-\left(A_{S}+A_{N}\right)=-\frac{2}{(\Delta z)^{2}}
\end{aligned}
$$

$$
B_{W}=\frac{2 r_{w}}{\left(\Delta r+2 r_{w}\right)(\Delta r)^{2}}
$$$$
B_{E}=\frac{2\left(r_{w}+\Delta r\right)}{\left(\Delta r+2 r_{w}\right)(\Delta r)^{2}}
$$

$$
B_{P}=-\left(B_{E}+B_{W}\right)=-\frac{2}{(\Delta r)^{2}}
$$

Since there are two unknown variables $\theta$ and $\phi$ in equation (13), $\phi$ is first transformed into $\theta$ by using equations (7)-(9) and then equation (13) which involves only one variable $\theta$ can be solved numerically. The eneregy equation for wall, equation(10), can be handled in a similar way. As for the PCM/wall interface, the harmonic mean procedure is adopted and the detail is described in [2]. It is noted that the finite difference equations for control volumes at the right and bottom boundaries are derived via the en- 


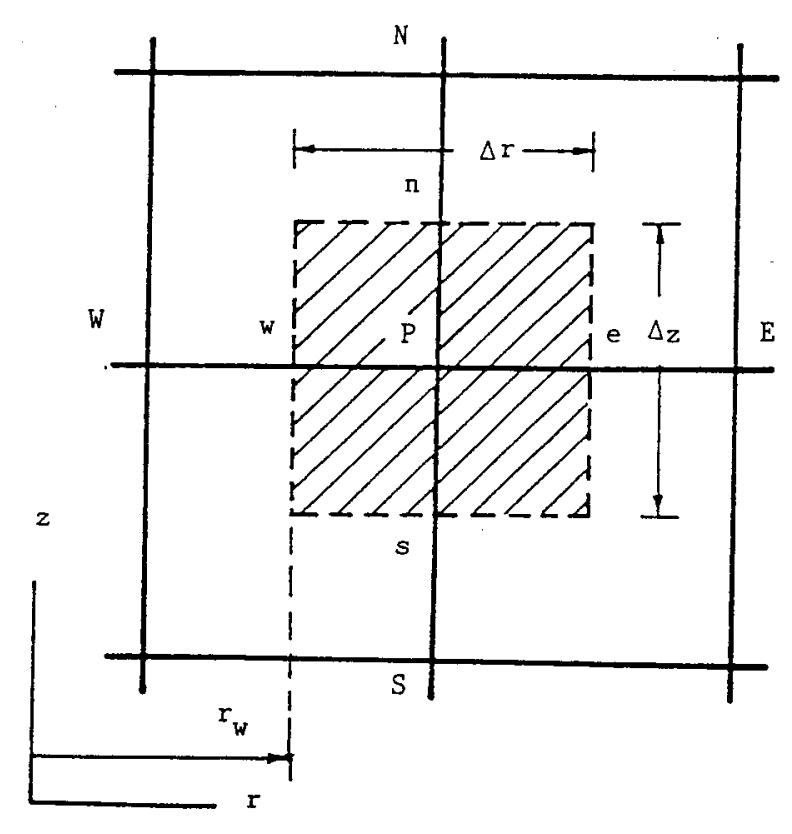

Fig. 2. Schematic diagram of a PCM control volume.

ergy balance concept. The derivation procedure can be also found in [2]. These two equations contain two important dimensionless parameters $B i_{\infty l}$ and $B i_{\infty 2}$. They are defined by the following equation

$$
B i_{\infty 1}=\frac{h_{\infty 1} \cdot R}{k_{w}} ; B i_{\infty 2}=\frac{h_{\infty 2} \cdot R}{k_{w}}
$$

In equation (15), $h_{\infty 1}$ and $h_{\infty 2}$ denote the heat transfer coefficients at the right and the bottom boundaries respectively. These Biot numbers are set to be equal to each other in this study for simplification.

The complete system of the discretized equations which cover the whole domain were solved by the ADI method. This method is second-order accurate in time and space and is unconditionally stable for the linear heat conduction problems. Although the solidification problem is nonlinear due to phase change, and therefore the ADI scheme is only conditionally stable for the present problem, however, the numerical experiments performed in the study show that the ADI scheme is still quite stable for the case with relatively large time step size. It should be mentioned that the tri-diagonal matrices are generated when applying the ADI scheme to solve the $2-D$ problems. Therefore, the well known Thomas algorithm can be employed, and hence the memories and the computational time can be reduced significantly.

Two important physical quantities are defined in this paper, which are fraction forzen $F F$, and dimensionless energy content $\bar{Q}$. For direct comparision, the definitions of these two quantities are the same as those in [15]. The fraction frozen $F F$ is defined as the ratio of the volume for the solid PCM to the total PCM volume. As to the dimensionless energy content $\bar{Q}$, its definition is the sum of the sensible energy and the latent heat for the PCM during the solidification process, divided by the total PCM energy before solidification. The results of the dimensionless energy content can clearly show the proportion of the total PCM energy released during the solidification process.

The iteration procedure for obtaining the solutions is described as follows. If some control volumes are solid i.e., $\phi<0$ at previous time, then $\phi$ is replaced by $\theta$ in the finite difference equation, equation (13). After solving the finite difference equation with the ADI method, if several control volumes are found to be at the fusion temperature, then the coefficients in equation (13) are set to be zero and $\phi$ is set equal to $\theta$. Consequently one more iteration is needed. If some of the control volumes are liquid, i.e., $\phi>0$, then the coefficients in equation (13) are multiplied by $\hat{c}$, and $\phi$ is still replaced by $\theta$. The rest procedure is the same as that described just above. The detailed information about such iteration procedure can be found in $[1,11,13]$.

\section{RESULTS AND DISCUSSION}

As aforementioned, this paper analyzes the solidification problem for a PCM in a cylindrical container. The effects of each controlling parameter are the main issues in this study. This section contains some important results and discussion as well as a comparision between the present results and the results from a previous study.

\section{Numerical Experiments and a Comparision with Previous Results}

Fig. 3 depicts the fraction frozen results for the case with isothermal boundary conditions on the right and bottom surfaces. Also shown are the results from Ref. [15]. Fig. 3 clearly shows that the agreement between the present results and the previous results is pretty good. This check is one of the important tasks in examining the accuracy of the other present results based on the convective boundary condtions. In addition, two more numerical experiments were performed in this study. First, the effects of the grid size were checked by using two different grids, $21 \times 60$ and $24 \times 72$. The difference was found to be quite small. Next the effects of the time step size were examined by performing several experiments. It was found that the influence of the time step size is negligible. However, in order to make the change for $\theta$ between two consecutive times not too large (less than $5 \%$, in 


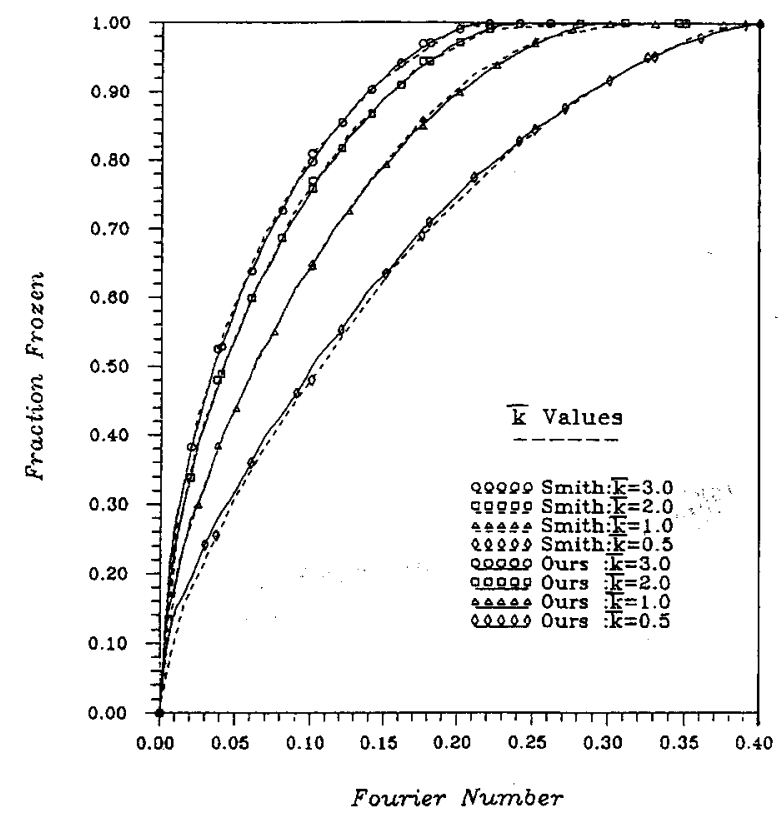

Fig. 3. A comparision between the present results and the results of [15].

general), the non-dimensional time step size was taken as $F_{o} \doteq 2.27 \times 10^{-5}$ in this study.

\section{Effects of the Biot Numbers}

Fig. 4 shows the effects of the Biot numbers when convective boundary conditions are considered on the right and the bottom boundaries. In Fig. 4 the Biot number varies from $10^{-2}$ to $10^{3}$. As expected, the solidification rate increases when the Biot number increases. Similarly, the dimensionless energy content decreases at a faster rate for larger Biot numbers. It should be noted that the results for the Biot number equal to $10^{3}$ almost fall on top of the curves based on the isothermal boundary conditions. Therefore, their results are expressed by only one curve. The above results clearly verify the theory that the convective boundary condition approaches the isothermal condition when the Biot number increases to infinity.

\section{Results and Discussion Based on Convective Boundary Conditions}

So far, the effects of the Biot number have been discussed in detail. These Biots numbers correspond to various cooling conditions. If the cooling medium is chosen to be air, then the corresponding Biot number can be found to be about 10 . Hence the results presented hereafter will be based on the Biot number of 10 . The values for the other parameters were chosen as follows. (1) For the PCM part, $\bar{R} \doteq 0.325$, $\bar{Z} \doteq 0.331, \bar{k}=2.0, \bar{G}=0.1, \quad \bar{\alpha}=2.0$, Ste $=1.0$, $\hat{c} \doteq 1.004$, (2) the initial values of $\phi$ and $\theta$ for PCM

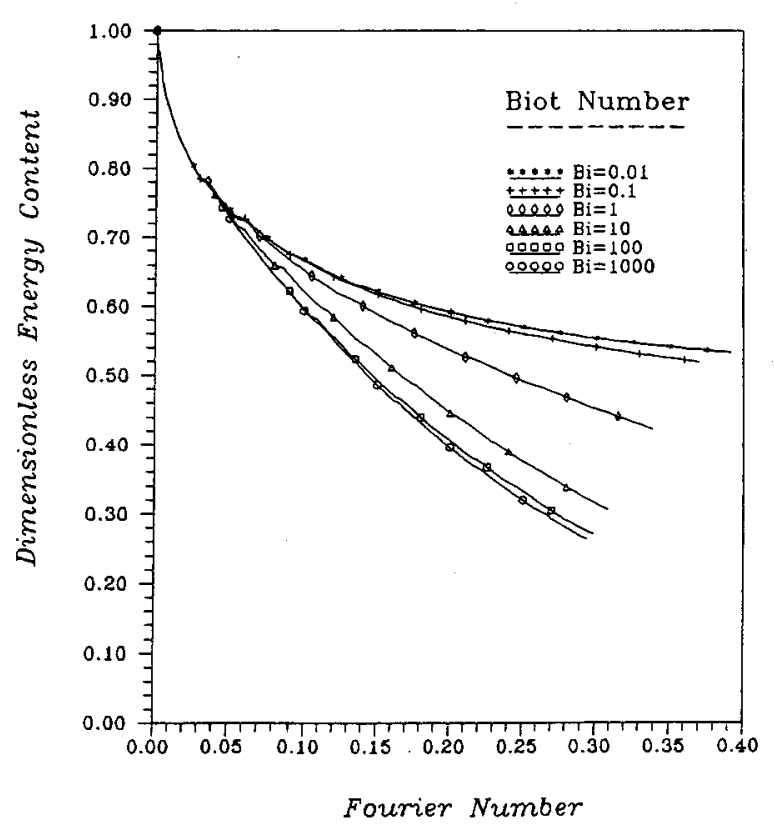

Fig. 4a Effects of the Biot number on the fraction frozen results.

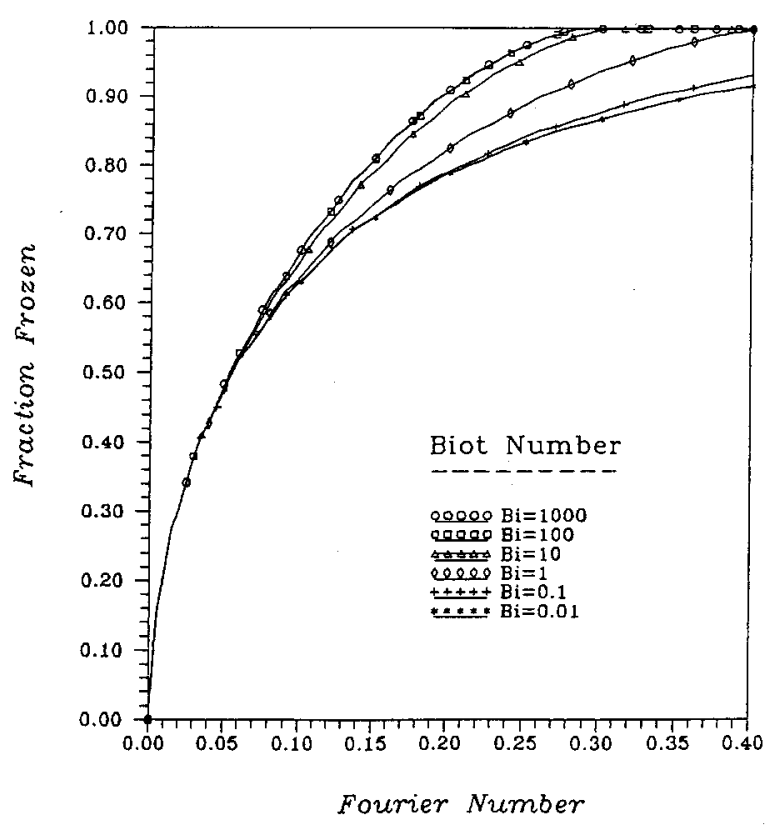

Fig. 4b. Effects of the Biot number on the nondimensional energy content.

were set to be 0 and 1.0 , respectively, and (3) the initial value of $\phi$ for wall was set to be -1.0 . Based on the standard values for the controlling parameters mentioned above, the influence of each parameter was studied systematically. The corresponding results are presented and discussed below.

(1) Effects of $\bar{k}$

$\bar{k}$ is the ratio of the thermal conductivity of wall 
to the thermal conductivity of solid PCM. The increase in $\bar{k}$ can be regarded as the increase in the thermal conductivity of wall if the thermal conductivity of solid PCM is taken to be a constant. Fig. 5 demonstrates clearly that as $\bar{k}$ increases, the solidification rate increases accordingly. The rate of release of the energy content increases also.

\section{(2) Effects of the Aspect Ratio}

$\bar{G}$ is definied as the ratio of the outer radius to the height of the container. Therefore, $\vec{G}$ is actually

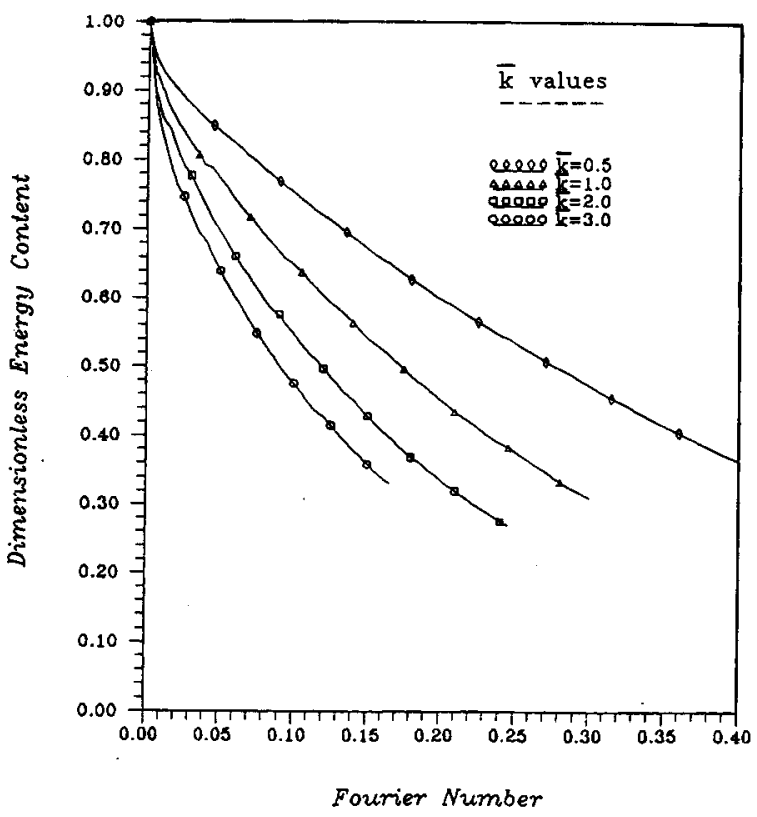

Fig. 5a. Effects of $\bar{k}$ on the fraction frozen results.

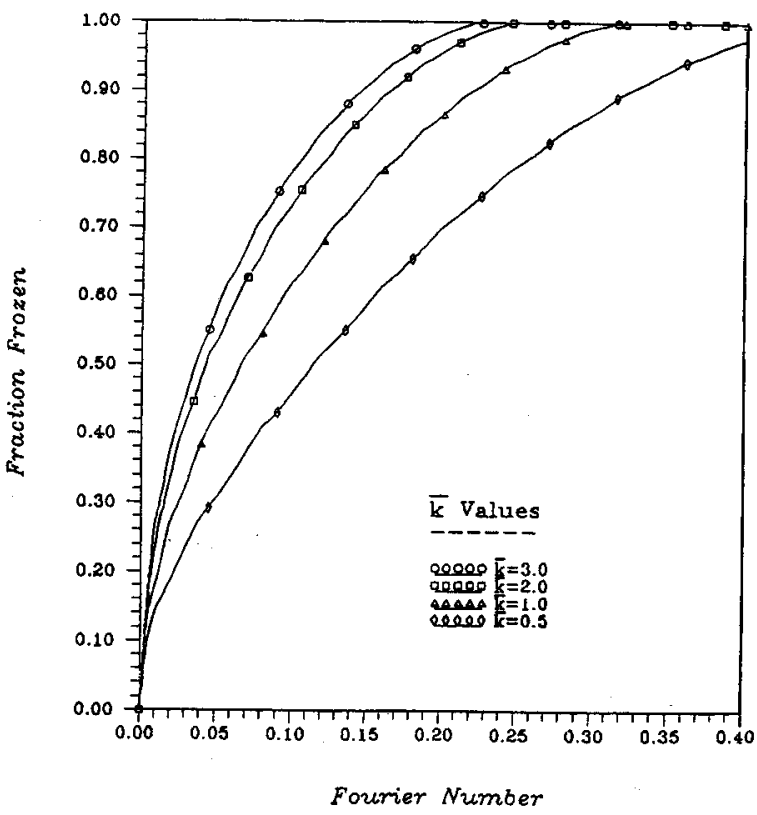

Fig. 5b. Effects of $\bar{k}$ on the nondimensional energy content. the inverse of the aspect ratio of the container. In the present study, different $\bar{G}$ values were obtained by varying the height of the container while keeping the outer radius unchanged. Fig. 6 shows that the solidification rate increases as $\bar{G}$ increases. This can be explained as follows. When $\vec{G}$ increases, i.e., the height decreases, the heat transfer area decreases accordingly, and the volume of the PCM of the container decreases also. After all, an increase in $\bar{G}$ causes a decrease in the ratio of the heat transfer area to the PCM volume, and finally leads to an increase in the solidification rate.

\section{(3) Effects of the Stefan Number}

According to the definition of the Stefan number, larger Stefan numbers correspond to larger temperature difference when the thermal properties of the PCM remain unchanged. Therefore, it is expected that as the Stefan number increases, the cooling rate and also the solidification rate for the PCM will increase. The results shown in Fig. 7 coincide with such expectation quite well.

\section{(4) Effects of the Wall Thickness}

Two parameters are related to the wall thickness, namely, $\bar{R}$ and $\bar{Z} . \quad \bar{R}$ is the ratio of the wall thickness in the radial direction to the outer radius of the container, and $\bar{Z}$ is the ratio of the wall thickness at the bottom to the height of the container. As $\bar{R}$ and $\bar{Z}$ decrease, the wall thickness decreases, and the vol-

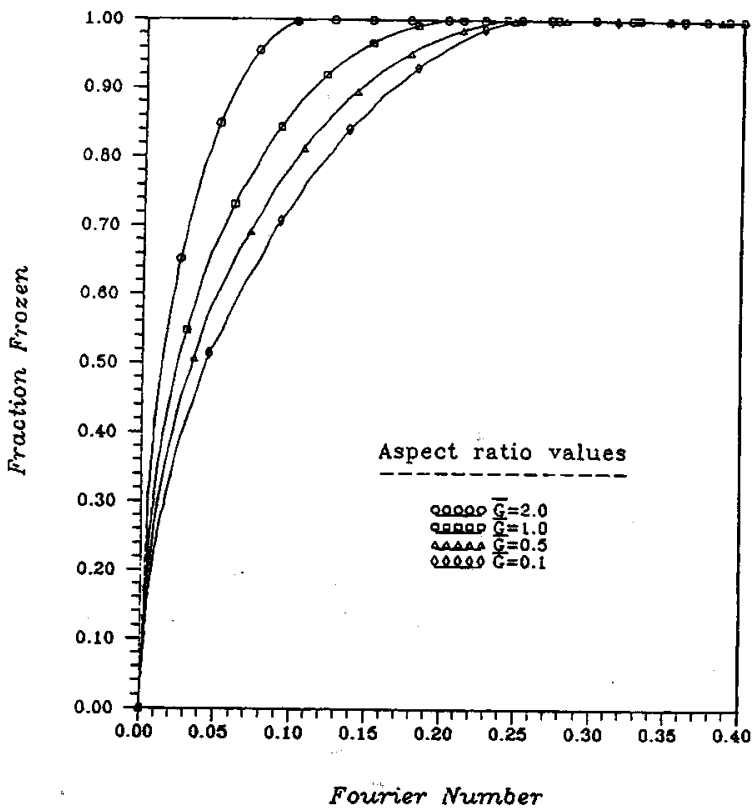

Fig. 6. Fraction frozen vs: Fourier number curves for different $\bar{G}$ values. 


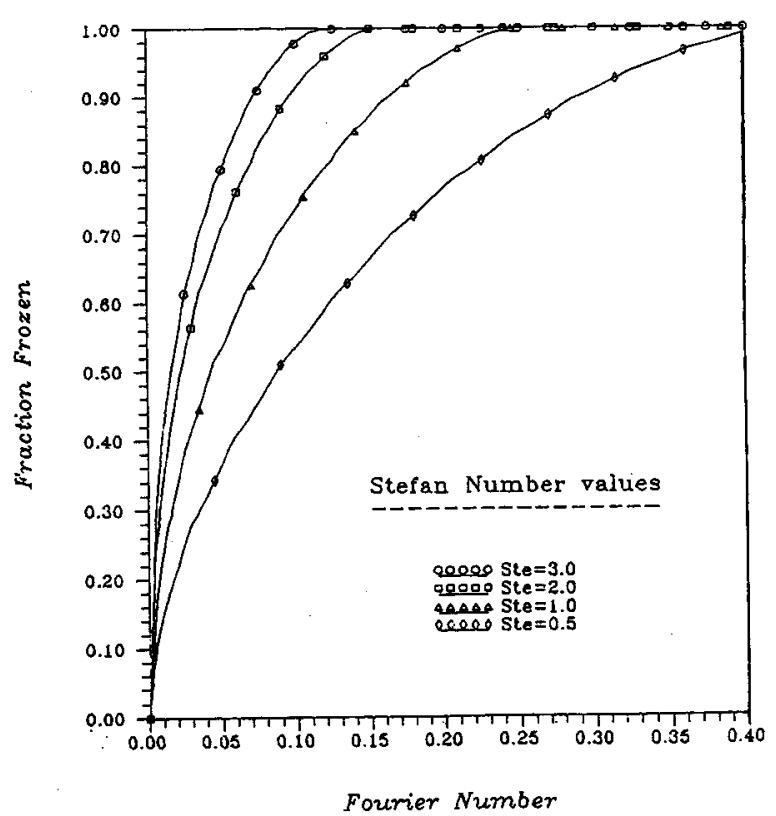

Fig. 7. Effects of the Stefan number on the fraction frozen results.

ume for the PCM will increase, provided that the outer radius $R$ and the height of the container $Z$ are fixed. However in the definition of the Fourier number, the reference length is taken to be the outer radius of the container $R$ rather than the radius of the PCM itself. Therefore, the abscissa of Fig. 8 is modified from Fo to $F_{0} /(1-\bar{R})^{2}$ for a meaningful comparision. Fig. 8 indicates that the solidfication rate increases as $\bar{R}$ and $\bar{Z}$ decrease, i.e., as the wall thickness decreases. This is due to the fact that a decrease in the wall thickness causes a decrease in the thermal resistance, and hence an increase in the heat tranefer rate as well as the solidification rate.

\section{(5) Effects of $\bar{\alpha}$}

$\bar{\alpha}$ is the ratio of the thermal diffusivity of the wall to that of the PCM. It can shown that

$$
\frac{\bar{k}}{\bar{\alpha}}=\frac{\rho_{w} c_{w}}{\rho c}
$$

Eq. (16) reveals that for $\bar{k}$ and $\rho \mathrm{c}$ fixed, the smaller $\bar{\alpha}$ is, the larger $\rho c$ (i.e., the heat capacity of the wall) will be. Since the initial temperature of the wall is lower than the PCM fusion temperature, a decrease in $\bar{\alpha}$ will enhance the cooling effect and therefore increase the solidification rate, as shown in Fig. 9. However, it should be pointed out that the difference among the results for different $\bar{\alpha}$ values is not so prominent since the external cooling conditions constitute the main body of the heat transfer modes in the present problem.

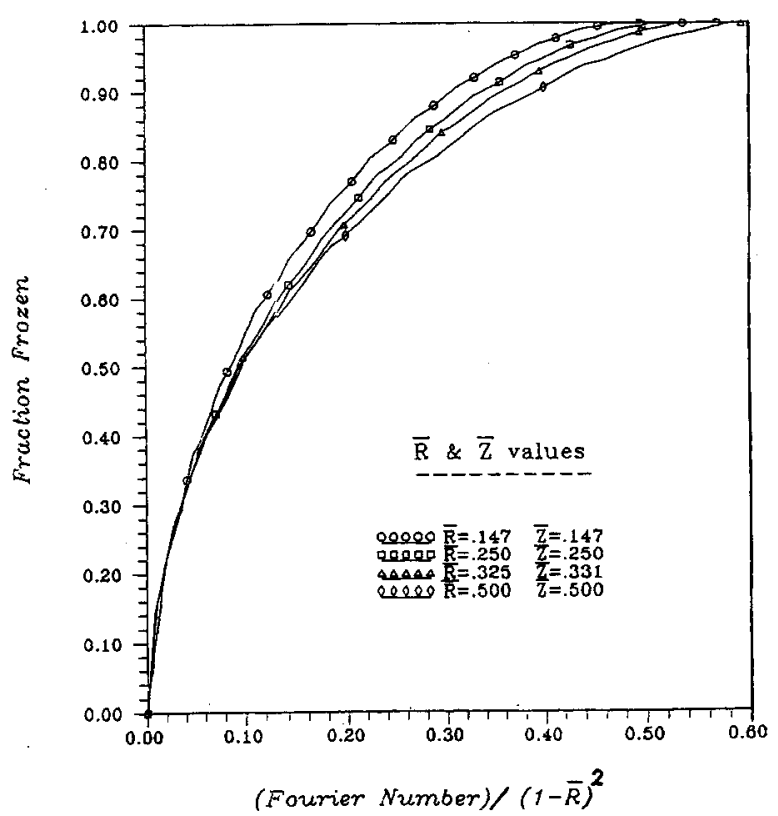

Fig. 8. Effects of the wall thickness on the fraction frozen results.

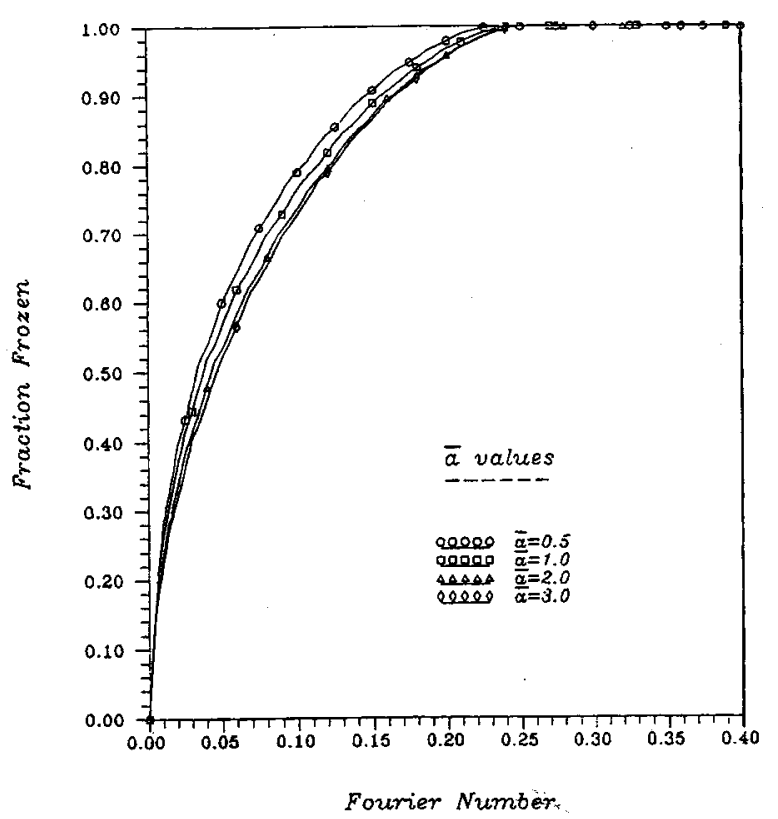

Fig. 9. Fraction frozen vs. Fourier number curves for differents $\bar{\alpha}$ values.

\section{(6) Movement of the Interface}

Up to now, the effects of each parameter, except the PCM initial temperature, have been analyzed in detail. All the results are presented in fraction frozen and the dimensionless energy content. However, the movement of the solid/liquid interface (i.e., phase front) is also of some interest. Fig. 10 depicts the movement of the phase front. It can be seen clearly that the PCM solidifies inwards as expected. It should 


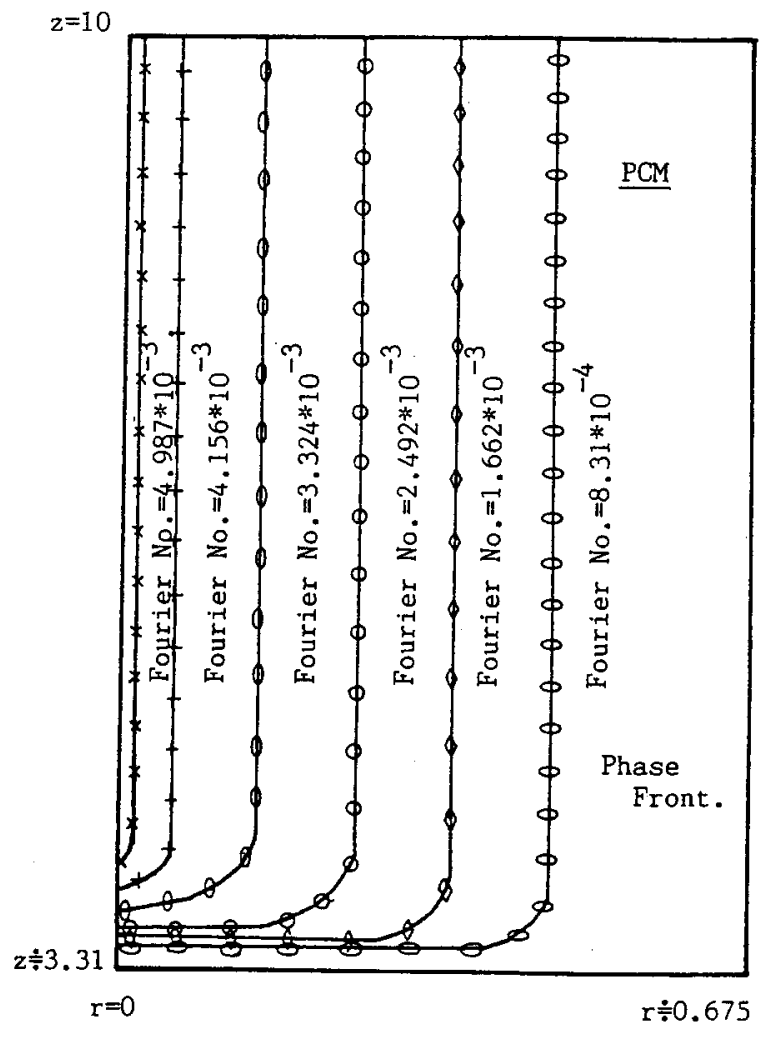

Fig. 10. Movement of the phase front.

be mentioned that Fig. 10 is based on the standard values for the controlling parameters.

\section{(7) Effects of Superheat for the PCM}

In all of the results presented above, the initial temperature of PCM was set to be at the PCM fusion temperature. Now, the efects of superheat of the PCM are studied. Fig. 11 displays the fraction frozen results, in which $\phi=0$ corresponds to the case without superheat, and $\phi=0.5$ and 1.0 correspond to the cases with superheat. It can be seen clearly that the solidfication rate is depressed due to superheat. It should be mentioned that as time elapses, the solidification rate for the case with superheat approaches that for the case without superheat gradually.

\section{CONCLUSIONS}

This paper analyzes the soldification problem for a PCM in a cylindrical container. The solution procedure is relatively simple and the numerical method employed is quite efficient. The effects of each controlling parameter are investigated systematically. The reults cam be concluded as follows.

(1) The external cooling conditions have a direct impact on the soldification process.

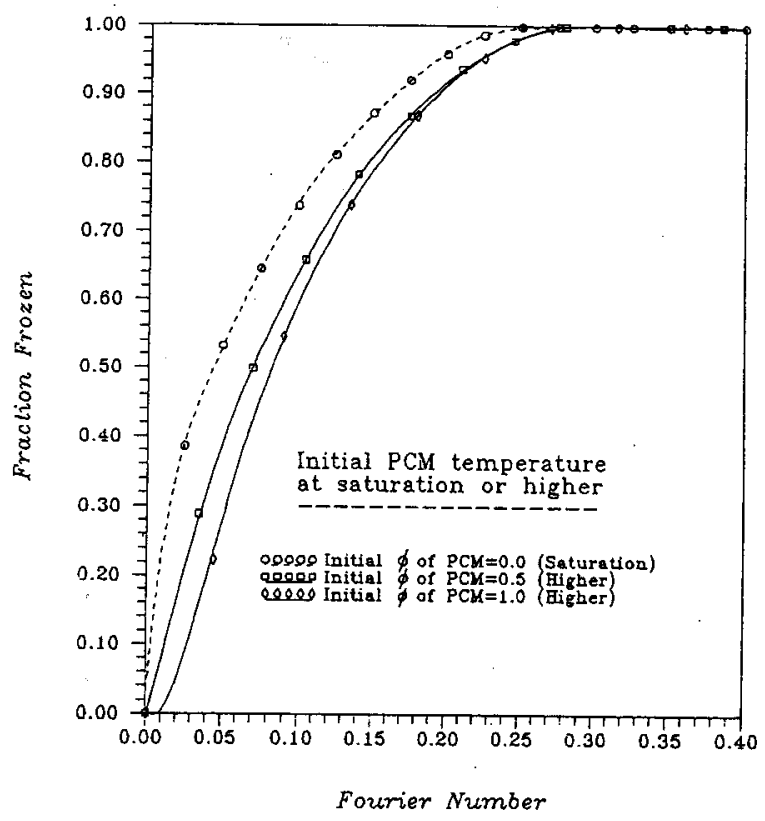

Fig. 11. Effects of superheat of PCM on the fraction frozen results.

(2) Under moderate cooling conditions, the influence of the controlling parameters including $\bar{k}$ ( the ratio of thermal conductivity of wall to that of solid PCM ), the dimensions of the container ( such as $\bar{G}, \bar{R}, \bar{Z}$ ) and the Stafen number on the soldification rate is quite significant. However, the influence of $\bar{\alpha}$ ( the ratio of thermal diffusivity of wall to that of solid PCM) is not so prominent.

(3) Superheat of PCM causes a decrease in solidfication rate, compared with the case without superheat. At later times, the difference becomes smaller.

\section{NOMENCLATURE}

\begin{tabular}{|c|c|}
\hline$A, B$ & coefficients in equation (13) \\
\hline$B i$ & Biot number \\
\hline$c$ & specific heat of solid PCM \\
\hline$c_{L}$ & specific heat of liquid PCM \\
\hline$\hat{c}$ & $\begin{array}{l}\text { ratio of the specific heat of solid PCM to } \\
\text { that of liquid PCM }\end{array}$ \\
\hline$F F$ & fraction frozen \\
\hline$F_{0}$ & $\begin{array}{l}\text { Fourier number and also nondimensional } \\
\text { time }\end{array}$ \\
\hline $\begin{array}{l}\Delta F_{o} \\
\bar{G}\end{array}$ & $\begin{array}{l}\text { nondimensional time step size } \\
\text { ratio of the outer radius to the height of the } \\
\text { container }\end{array}$ \\
\hline$h$ & enthalpy \\
\hline$\underline{k}$ & thermal conductivity \\
\hline & $\begin{array}{l}\text { ratio of the thermal conductivity of wall to } \\
\text { that of solid PCM }\end{array}$ \\
\hline $\bar{Q}$ & dimensionless energy content \\
\hline
\end{tabular}


$r \quad$ nondimensional radial coordinate

$\Delta r \quad$ nondimensional grid size in the radial direction

$r_{w}$ radial component of the distance between the origin and the west face of control volume $P$

$R \quad$ outer radius of the container

$R_{w} \quad$ radial wall thickness of the container

$\bar{R} \quad R_{w} / R$

Ste Stefan number

$t \quad$ time

$T \quad$ temperature

$T_{\text {ref }} \quad$ reference temperature

$T_{w} \quad$ wall temperature

$z$

$\Delta z$

nondimensional vertical coordinate

nondimensional grid size in the vertical direction

$Z \quad$ ight of the container

$Z_{w} \quad$ wall thickness of the container at the bottom

\section{$\bar{Z} \quad Z_{w} / Z$}

$\alpha \quad$ thermal diffusivity

$\bar{\alpha} \quad$ ratio of the thermal diffusivity of wall to that of solid PCM

$\gamma \quad$ radial coordinate

$\Delta \gamma \quad$ grid size in the radial direction

$\zeta \quad$ vertical coordinate

$\theta$ nondimensional enthalpy

$\lambda$ latent heat of fusion of PCM

$\rho$ density

$\phi \quad$ nondimensional temperature

\section{Subscripts}

\section{fr saturated state \\ $L, l \quad$ liquid phase of PCM}

$N, S, E, W$ grids located at the top, bottom, right and left sides of the control volume, respectively

$P \quad$ central point of the control volume

$s \quad$ solid phase of PCM

$v \quad$ quantities referred to wall

$\infty \quad$ ambint

\section{REFERENCES}

1. Bonacina, C., Comini, G., Fasano A., and Primicerio, M., "Numerical Solution of PhaseChange Problems," Int. J. Heat Mass Transfer, Vol. 16, pp. 1825-1832 (1973).

2. Chang, J.S., "A Study of Solidification in a Cylindrical Container," M.S. Thesis, National Taiwan Ocean University, Keelung, Taiwan (1992).

3. Chiou, H.S., Chu, M.T., Yen, R.H., and Pan, E.N.,"Analysis of Solidification Pattern of the Al-
Alloy in Step-plate Castings," 6th. Nat. Conf. on Mech. Eng. CSME, pp. 563-573 (1989).

4. Chu, M,T., Chiou, H.S., Yen, R.H., and Pan, E.N.,"Analysis of Solidification Pattern for BossShaped Castings," 5th. Nat. Conf. on Mech. Eng. CSME, pp. 617-628 (1988).

5. Fisher, K.M., "The Effects of Fluid Flow on the Solidification of Industrial Castings and Ingots," Physico Chemical Hydrodynamics, Vol. 2, pp. 311-326, (1981).

6. Kattamis, T.Z., "Heat Transfer and Mass Transfer During Solidification," in: Heat and Mass Transfer in Metallurgical Systems, D.B. Spalding and N.H. Afgan, eds., Hemisphere, Washington, pp. 375- 401 (1981).

7. Lee, S.L., and Tzong, R.Y., "An Enthalpy Formulation for Phase Change Problems with a Large Thermal Diffusivity Jump across the Interface," Int. J. Heat Mass Transfer, Vol. 34, pp. 1491-1502 (1991).

8. Patankar, S.V., "Numerical Heat Transfer and Fluid Flow", pp. 44-47 (1980).

9. Ramachandran, N, Gupta, J.P., and Jaluria, Y, "Thermal and Fluid Flow Effects during Solidification in a Rectangular Enclosure," Int. J. Heat Mass Trnsfer, Vol. 25, pp. 187-194 (1982).

10. Ramachandran, N, Gupta, J.P., and Jaluria, Y., "Experiments on Solidification with Natural Convection in a Rectangular Enclosed Region," Int. J. Heat Mass Transfer, Vol. 25, pp. 595-596 (1982).

11. Raw, M.J., and Schneider, G.E., "A New Implicit Solution Procedue for Multidimensional FiniteDifference Modeling of the Stefan Problem," J. Energy, Vol. 7, pp. 652-659 (1983).

12. Schneider, G.E., "A Numerical Study of Phase Change Energy Transport in Two-Dimensional Rectangular Enclosures," J. Energy, Vol. 7, pp. 652-659 (1983).

13. Schneider, G.E., and Raw, M.J., "An Implicit Solution Procedure for Finite Difference Modeling of the Stefan Problem," AIAA J., Vol. 22, pp. 1685-1690 (1984).

14. Shamsundar N, and Sparrow, E.M., "Analysis of Multidimensional Conduction Phase Change Via the Enthalpy Model," J. Heat Transfer, Vol. 97, pp. 333-340 (1975).

15. Smith, R.N., Pike, R.L., and Bergs, C.M., "Numerical Analysis of Solidification in a ThickWalled Cylindrical Container," J. Thermophysics, Vol. 1, pp. 90-96 (1987).

16. Sparrow, E.M., and Broadbent, J.A., "Freezing in a Vertical Tube," J. Heat Transfer, Vol. 105, pp. 217-225 (1983).

17. Sparrow, E.M., and Ohkubo, Y., "Numerical Analysis of Two Dimensional Transient Freezing Including Solid-Phase and Tube-Wall Conduc- 
tion and Liquid-Phase Natural Convection," Num.

Heat Transfer, Vol. 9, pp. 59-77 (1986).

18. Sparrow, E.M., and Ohkubo, Y.,"Numercial Predictions of Freezing in a Vertical Tube," Num. Heat Transfer, Vol.9, pp.79-95 (1986).

19. Viskanta, R, "Heat Transfer During Melting and Solidification of Metals," J. Heat Transfer, Vol. 110, pp. 1205-1219 (1988). 